\title{
Is the quantitative pain assessment method "Pain Degree" less susceptible to psychological factors than VAS?
}

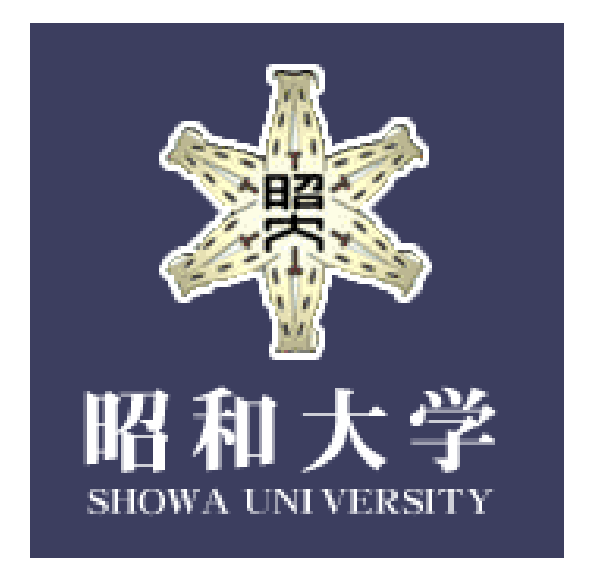

Okayasu T. ${ }^{1}$, Hasegawa Y. ${ }^{1}$, Mera H. ${ }^{2}$, Kobayashi Y. ${ }^{2}$, Ichimura M. ${ }^{3}$, Otake H. ${ }^{3}$

1Showa University Fujigaoka Hospital, Dept of Anaesthesiology, Yokohama, Japan,

2Tokyo Metropolitan Health and Medical Treatment Corporation Ebara Hospital,

Dept of Anaesthesiology, Tokyo, Japan,

${ }^{3}$ Showa University Hospital, Dept of Anaesthesiology, Tokyo, Japan

Background and Goal of Study

Although Visual Analogue Scale (VAS) is widely used, VAS is considered to be affected by a patient's mental state. We can use the perception/pain quantitative analytical device and obtain as values of Pain Degree (PD) in Japan. Many patients show that VAS and PD tend to decrease together after treatment, but some patients do not so. We examined whether the quantitative pain assessment method "PD" is affected psychologically using the results of psychological tests.

\section{Materials and Methods}

After approval of the institutional ethics committee, 52 adult patients (27-90 y.o.) suffering from chronic pain were candidates for this study. Subjective evaluations of pain were VAS.

Objective evaluations of pain were measured by a electrical neurostimulator (Pain vision ${ }^{\mathrm{TM}}$, Nipro, Japan).

It delivers a sinusoidal constant alternating current, and measure different types of the current perception threshold (CPT).

One CPT is the minimum perception threshold (MPT) .

The other CPT (PET) is the current threshold which the patient perceives the equivalent strength as the chronic pain.

PD was calculated: (PET - MPT)/MPT.

We measured VAS and PD at pre- and post - treatment.

As psychological test, we used Kyushu Medical Index questionnaire which simplified Cornell Medical Index. We evaluated the number of the positive answers to $\mathrm{KMI}$ and compared the distribution from the number of the positive answers of the physical and the bodily appeal.

T-test was used for comparison pre- and post - treatment. Chi-square test was used for a comparison of the distribution. A value of $P<0.05$ was considered significant.

\section{Results}

There were significant changes of VAS

(58+/-25.4vs.33.7+/-27mm, mean+/-SD, p<0.00001) (Fig-1) , and PD (230.6+/-258.3 vs. 102.7+/-175.2, $\mathrm{p}=0.00012)($ Fig-2) between pre and post - treatment in total.

35 patients $(67 \%)$ revealed that VAS and PD tend to decrease together after treatment. There was not significant difference in the average of the positive answers between the group that VAS and PD tend to decrease together and the other group (21.3+/-13.5vs. $21.9+/-13.4 \mathrm{P}=0.88)$.

Fig-3 shows distribution of the number of the positive answers of both groups.

Discussion

There was not significant difference in the average of the positive answers between groups.

The distribution of the number of the positive answers of two groups are similar. (not significant)

Our study could find that PD was also affected psychologically as well as VAS.

Combination of conventional pain rating methods (e.g. VAS, Numerical Rating Scale) with assessment using PainVision enables multifaceted assessment of pain.

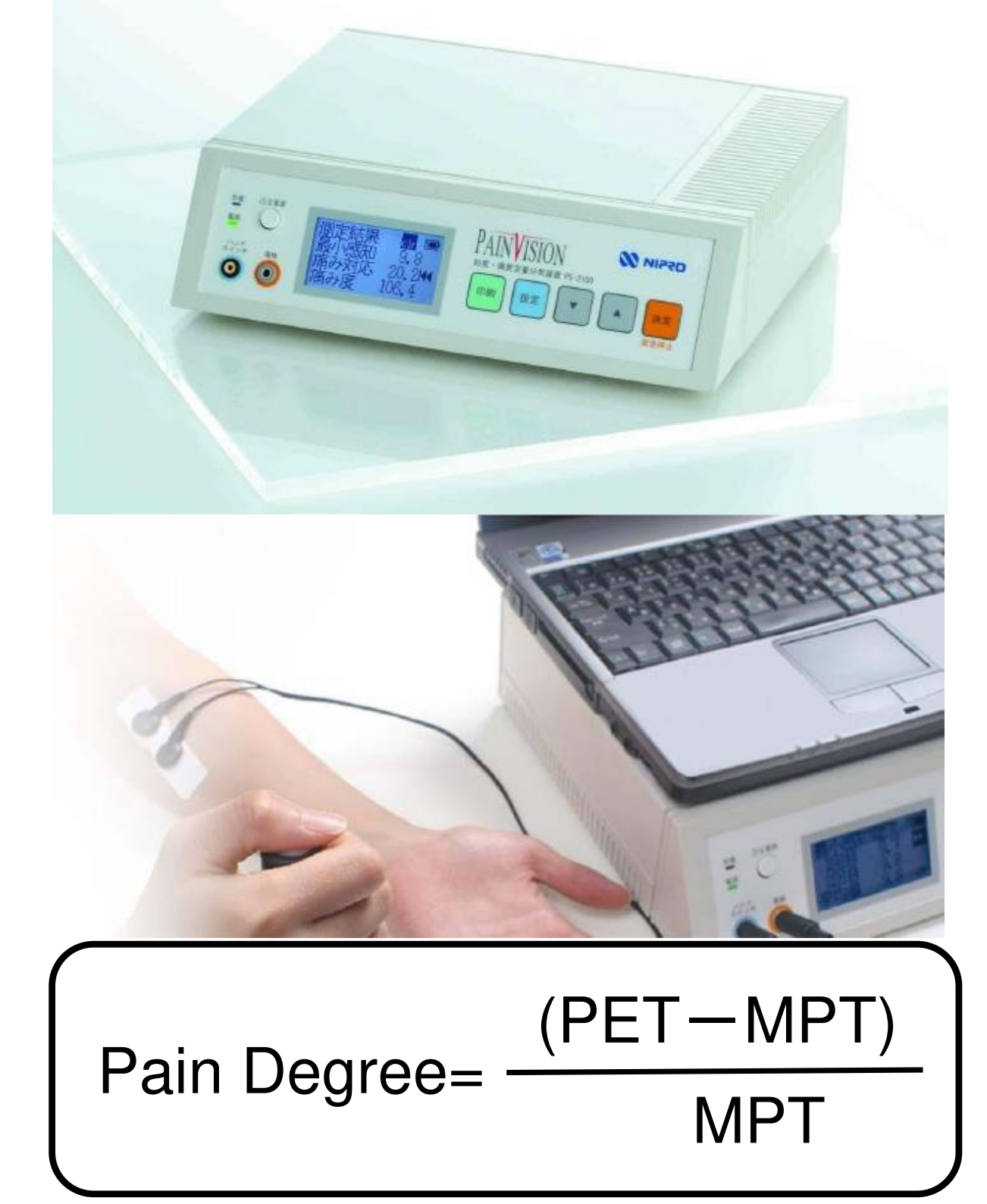

Changes of VAS and PD between pre and post - treatment 100 80 60 0 Fig.-1 pre

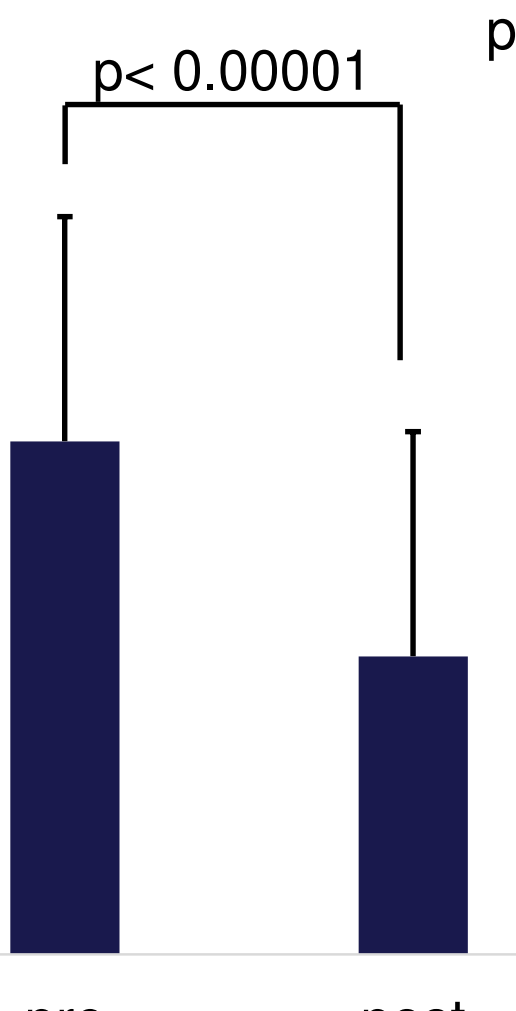

post

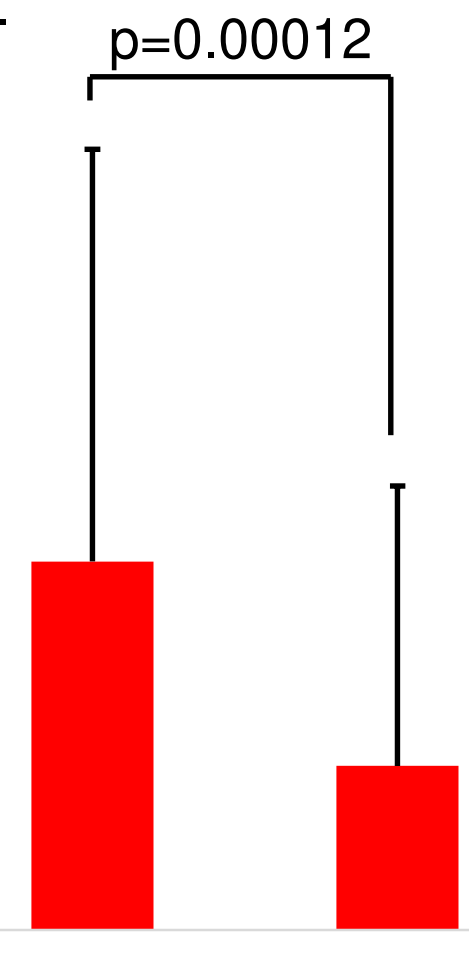

Fig.-2

post

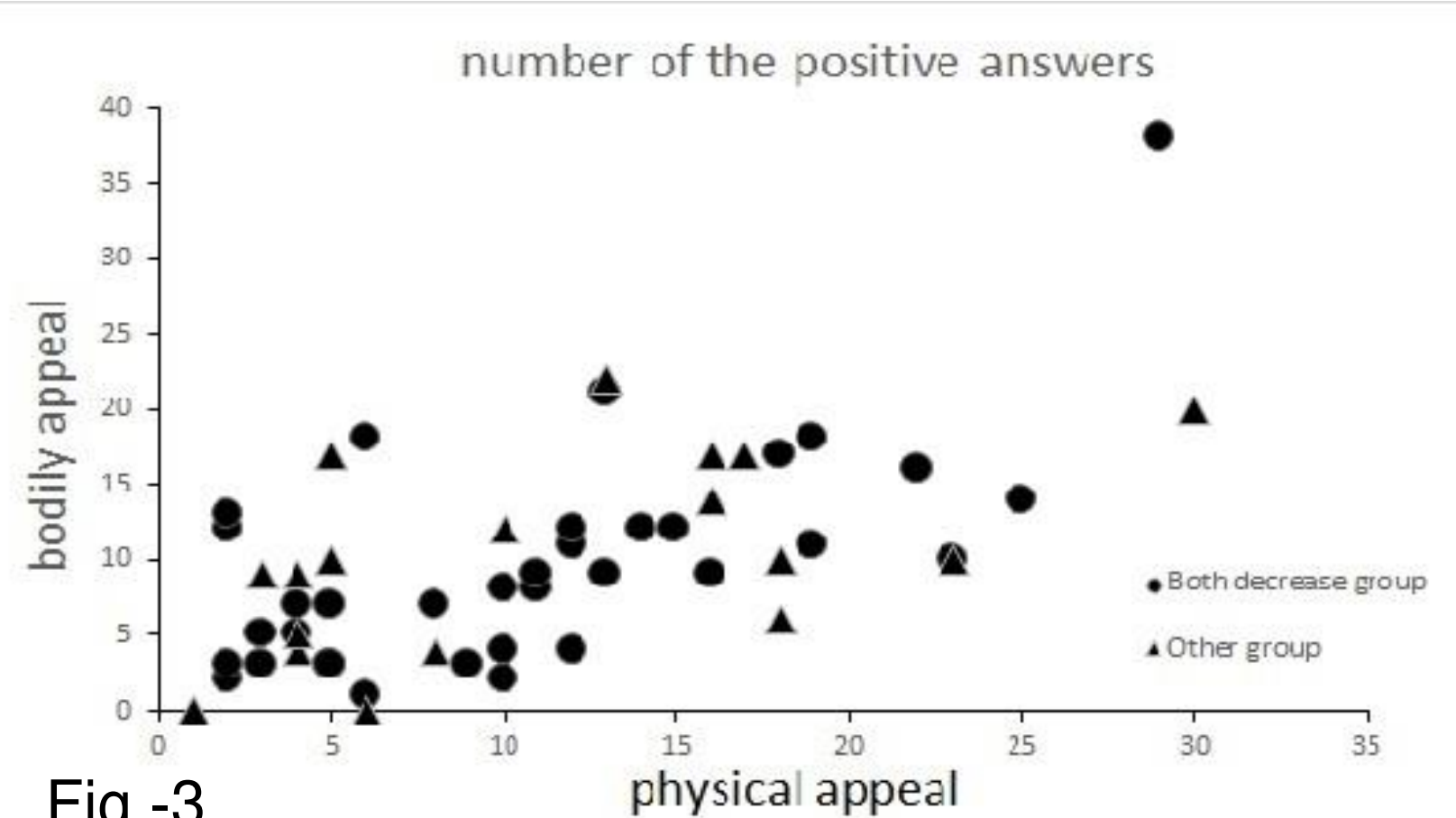

Fig.-3

physical appeal 